\title{
Observation of Weak Collapse in a Bose-Einstein Condensate
}

\author{
Christoph Eigen, Alexander L. Gaunt, Aziza Suleymanzade, Nir Navon, Zoran Hadzibabic, and Robert P. Smith* \\ Cavendish Laboratory, University of Cambridge, J. J. Thomson Avenue, \\ Cambridge CB3 OHE, United Kingdom
}

(Received 1 September 2016; published 19 December 2016)

\begin{abstract}
We study the collapse of an attractive atomic Bose-Einstein condensate prepared in the uniform potential of an optical-box trap. We characterize the critical point for collapse and the collapse dynamics, observing universal behavior in agreement with theoretical expectations. Most importantly, we observe a clear experimental signature of the counterintuitive weak collapse, namely, that making the system more unstable can result in a smaller particle loss. We experimentally determine the scaling laws that govern the weak-collapse atom loss, providing a benchmark for the general theories of nonlinear wave phenomena.
\end{abstract}

DOI: 10.1103/PhysRevX.6.041058

\section{INTRODUCTION}

Wave collapse occurs in a wide range of physical contexts, including optics and atomic and condensed-matter physics. Generally, collapse occurs if an attractive nonlinearity exceeds a critical value. If the collapse is triggered at time $t=0$, the wave amplitude asymptotically diverges at some point in space as the collapse time $t_{\mathrm{c}}$ is approached. In practice, the amplitude divergence results in dissipation of wave energy (or particle loss).

The unifying theoretical framework for understanding different collapse phenomena is provided by the nonlinear Schrödinger equation, which has been extensively studied for various forms of nonlinearity [1,2]. This general formalism is applied to self-focusing of light [3-7], collapse of Langmuir waves [8,9], and Bose-Einstein condensates (BECs) [10-14], as well as to surface water waves $[15,16]$.

In this framework, wave collapse is classified as either strong or weak (see Fig. 1). In a strong collapse, a finite fraction of the wave collapses towards the singularity. On the other hand, in a weak collapse [17-19], the fraction of the wave that (in the absence of dissipation) ultimately reaches the singularity vanishes. This has the counterintuitive practical implication that making the system more unstable, by quenching the nonlinearity further beyond the critical point, can result in less dissipation [19,20]. Qualitatively, once the collapse is triggered, for stronger attractive interactions, it happens faster and progresses further before dissipative processes halt it; consequently,

"rps24@cam.ac.uk

Published by the American Physical Society under the terms of the Creative Commons Attribution 3.0 License. Further distribution of this work must maintain attribution to the author(s) and the published article's title, journal citation, and DOI.
Subject Areas: Atomic and Molecular Physics, Interdisciplinary Physics, Nonlinear Dynamics

the wave amplitude is larger at the point in time when dissipation occurs, and for weak collapse, this means that a smaller fraction of the wave is actually dissipated. To our knowledge, weak collapse has not been experimentally observed in any physical system.

An atomic BEC with $s$-wave two-body interactions is modeled by the Gross-Pitaevskii (GP) equation, with a cubic nonlinearity proportional to the scattering length $a$, which can be dynamically tuned via a Feshbach resonance [21]. The BEC is prone to collapse for any $a<0$, but a kinetic-energy barrier makes it metastable up to a critical interaction strength [10-14]. If the BEC becomes unstable and collapses, dissipation occurs through three-body recombination that results in particle loss. Importantly, the three-dimensional GP equation is expected to provide an example of weak collapse.

Previous collapse experiments with atomic BECs [22-27] (see also Ref. [28]) were performed in the traditional setting of a harmonic trap. The critical point [23] and collapse times $[24,26]$ were in general agreement with

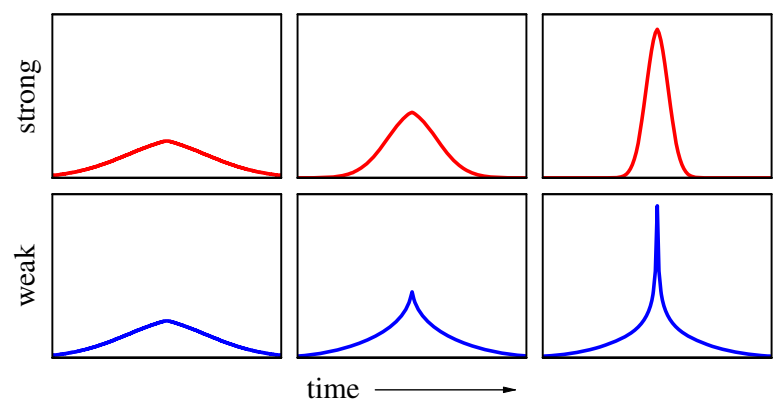

FIG. 1. Strong versus weak collapse (cartoon). In strong collapse, a finite portion of the wave (here $100 \%$, for simplicity) collapses towards the singularity. In weak collapse, as time progresses, a diminishing fraction of the wave approaches the singularity, with long tails left behind. 
theoretical expectations [10,36-43], but no evidence of weak collapse was observed; the atom loss was only seen to grow with $|a|$ [25].

In this article, we study BEC collapse in a new experimental setting, using a ${ }^{39} \mathrm{~K}$ condensate $[44,45]$ prepared in the uniform potential of an optical-box trap [46]; for details of our setup, see Appendix A. The combination of large system size (up to $41 \mu \mathrm{m}$ ) and fine-tuning of the scattering length (with a resolution of $0.03 a_{0}$, where $a_{0}$ is the Bohr radius) gives us a very large dynamic range: We observe metastable attractive BECs with up to $2 \times 10^{5}$ atoms and collapse times that vary between 3 and $400 \mathrm{~ms}$. We demonstrate the expected scaling of the critical scattering length $a_{\mathrm{c}}$ with the BEC atom number $N$ and the system size $L$, and show that the collapse time can be expressed as a universal function of the dimensionless interaction strength $a N / L$. Most importantly, we observe conclusive evidence for weak collapse, namely, the counterintuitive decrease of the atom loss with increasing $|a|$, and experimentally determine the scaling laws that govern the weak-collapse atom loss. The weak nature of the collapse is directly revealed only by resolving single collapse events, and it is obscured in the multiplecollapse regime, which has been seen in previous cold-atom experiments.

In Secs. II-IV, we address the following in turn: (i) the critical point for the collapse, (ii) the collapse dynamics in a system that is suddenly made unstable by an interaction quench, and (iii) the aftermath of the collapse, which reveals its weak nature.

\section{CRITICAL POINT}

The starting point for our discussion is the GP equation for a homogeneous box potential, with a heuristically added three-body loss term [13]:

$$
i \hbar \frac{\partial \psi}{\partial t}=-\frac{\hbar^{2}}{2 m} \nabla^{2} \psi+\frac{4 \pi \hbar^{2} a}{m}|\psi|^{2} \psi-i \frac{\hbar K_{3}}{2}|\psi|^{4} \psi
$$

where $m$ is the atom mass, $K_{3}$ is the three-body loss coefficient, $\psi$ is normalized to the atom number $N$, and the boundary condition is $\psi=0$ at the trap walls.

We use a cylindrical box trap [46] of variable length $L$ and radius $R$, and always set $R=L / 2$, so $L$ is the only length scale characterizing the system size. We may thus rewrite Eq. (1) in a dimensionless form, defining $\tilde{\mathbf{r}}=\mathbf{r} / L$ and $\tilde{t}=t / \tau_{0}$, with characteristic time $\tau_{0}=2 m L^{2} / \hbar$ :

$$
i \frac{\partial \tilde{\psi}}{\partial \tilde{t}}=-\nabla^{2} \tilde{\psi}+\alpha|\tilde{\psi}|^{2} \tilde{\psi}-i \eta|\tilde{\psi}|^{4} \tilde{\psi}
$$

where

$$
\alpha=\frac{8 \pi a N}{L} \quad \text { and } \quad \eta=\frac{N^{2} m K_{3}}{\hbar L^{4}}
$$
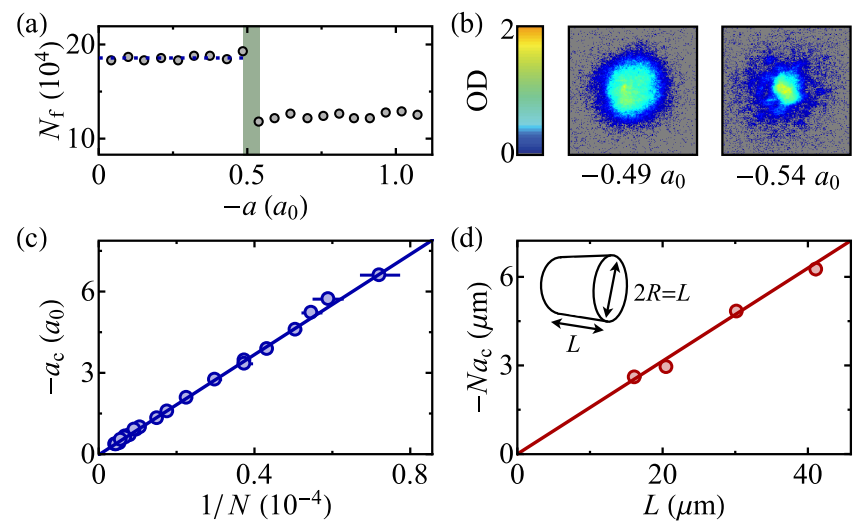

FIG. 2. Critical point for collapse. (a) Final atom number, $N_{\mathrm{f}}$, after ramping to a variable negative $a$, for $L=30(1) \mu \mathrm{m}$ and initial $N=18.7(5) \times 10^{4}$. The critical scattering length $a_{\mathrm{c}}$ is seen in the sharp drop in $N_{\mathrm{f}}$. (b) ToF images taken on either side of the critical point. (c) Variation of $a_{\mathrm{c}}$ with $N$, for $L=30 \mu \mathrm{m}$. The linear fit confirms the expected scaling $a_{\mathrm{c}} \propto 1 / N$. (d) Variation of $N a_{\mathrm{c}}$ with $L$. The linear fit confirms the scaling $N a_{\mathrm{c}} \propto L$.

and $\tilde{\psi}$ is initially normalized to unity. For the range of scattering lengths that we study, we assume that $K_{3}$ is constant $[47,48]$, with the value $1.3(5) \times 10^{-41} \mathrm{~m}^{6} \mathrm{~s}^{-1}$ [49]. The corresponding value of $\eta$, for all our $L$ and $N$ values, is very small $\left(<6 \times 10^{-4}\right)$, and thus three-body loss is negligible in our (meta)stable condensates. However, if the BEC collapses, significant loss occurs, providing the primary experimental signature of the collapse.

Neglecting the atom loss in a metastable BEC, based on Eq. (2), the critical interaction strength $\alpha_{\mathrm{c}}$ can depend only on the boundary conditions, i.e., the box shape. For a family of self-similar boxes $(R / L=$ const), it should be a universal constant, so $a_{\mathrm{c}} \propto L / N$.

To experimentally study the critical point for collapse, we prepare a stable BEC at $4 a_{0}$; then over $1 \mathrm{~s}$, we ramp the scattering length to a variable $a<0$ and wait for $2 \mathrm{~s}$ before turning off the trap and imaging the atoms after $100 \mathrm{~ms}$ of time-of-flight (ToF) expansion. We image the cloud along the axial direction of our cylindrical trap, and for ToF we jump the scattering length to $20 a_{0}$.

In Fig. 2(a), we show how, for a given initial $N$, the final atom number depends on the negative $a$. A well-defined $a_{\mathrm{c}}$ is signaled by a sharp drop in the atom number. As shown in Fig. 2(b), the atom loss is accompanied by a qualitative change in the appearance of the cloud in ToF.

In Fig. 2(c), we plot $a_{\mathrm{c}}$ for $L=30 \mu \mathrm{m}$ and a wide range of $N$ values, from $10^{4}$ to $2 \times 10^{5}$. We clearly observe the expected scaling $a_{\mathrm{c}} \propto 1 / N$ (see also Appendix B). In Fig. 2(d), we plot the measured $N a_{\mathrm{c}}$ versus box size and confirm the scaling $N a_{\mathrm{c}} \propto L$. We find that the dimensionless critical interaction strength is $\alpha_{\mathrm{c}}=-4(1)$, where the error includes the systematic uncertainties in box size and absolute atom number calibration. For comparison, numerical simulations of the GP equation for our box geometry give $\alpha_{\mathrm{c}}=-4.3$. 


\section{COLLAPSE DYNAMICS}

To study the collapse dynamics, we perform interactionquench experiments [24]. We prepare a BEC just above $a_{\mathrm{c}}$ and then quench the scattering length to a variable $a<a_{\mathrm{c}}$ to initiate the collapse (see Appendix B for more details). After a variable hold time $t$, we jump the scattering length to $20 a_{0}$, switch off the trap, and observe the cloud in ToF.

As shown in the left panel of Fig. 3(a), for quenches close to the critical point (small $\left|a-a_{\mathrm{c}}\right|$ ), at $t_{\mathrm{c}}$ the atom number suddenly drops to a stable lower value. We

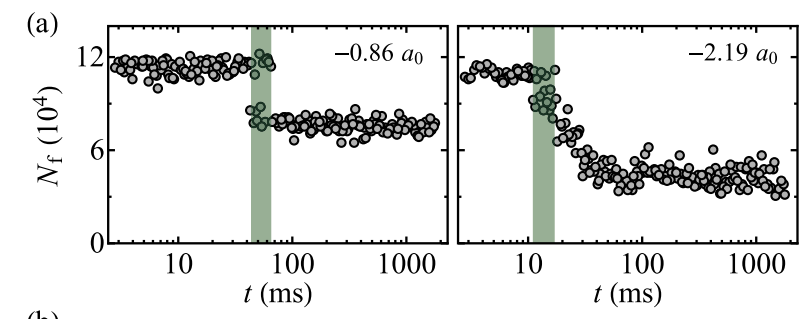

(b)
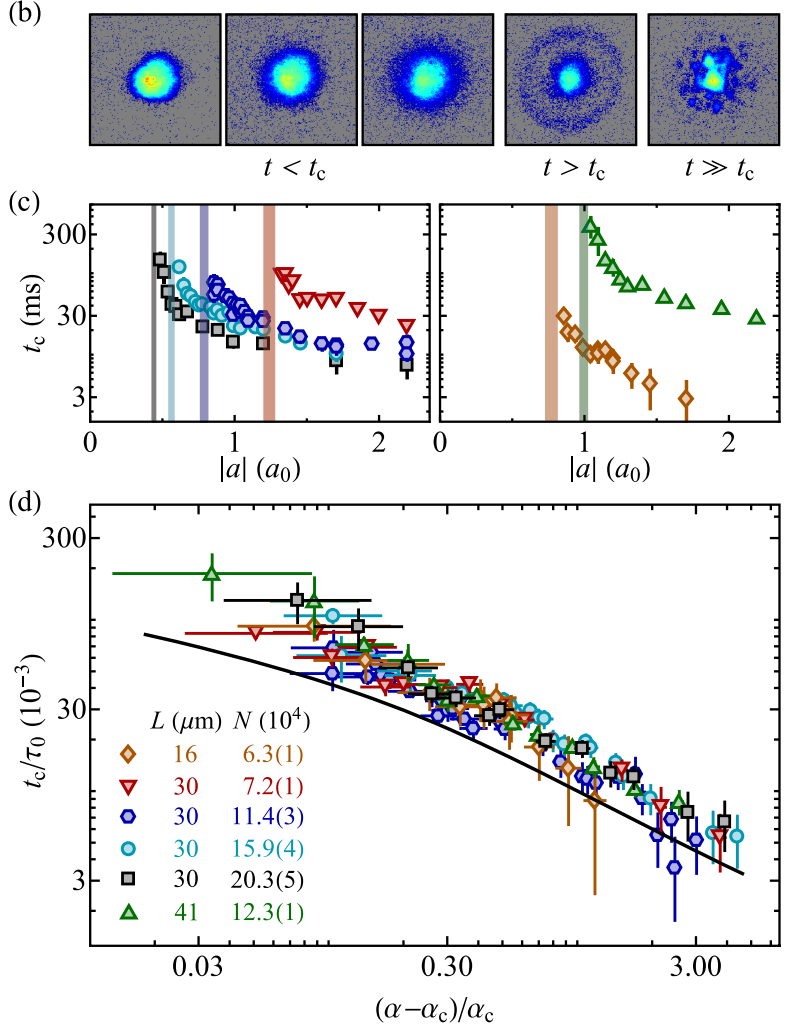

FIG. 3. Collapse dynamics. (a) Atom number versus time after quenches to $a=-0.86 a_{0}$ (left panel) and $-2.19 a_{0}$ (right panel); here, $L=30 \mu \mathrm{m}$ and $N=11.4 \times 10^{4}$, corresponding to $a_{\mathrm{c}}=-0.79 a_{0}$. Green bands indicate $t_{\mathrm{c}}$ and its uncertainty. (b) Typical ToF images at various stages after the quench (here, for $a=-1.02 a_{0}$ ). (c) Collapse time versus $a$ for six data sets taken for various $L$ and $N$; see legend in (d). The shaded bands indicate $a_{\mathrm{c}}$ values. (d) Universal collapse dynamics. We plot the dimensionless collapse time versus the reduced distance from the critical point, for all six data sets. The solid line shows the results of lossless GP simulations without any free parameters. understand this as a single collapse event. On the other hand, for large quenches [right panel of Fig. 3(a)], the atom number appears to gradually decay until it stabilizes. Such behavior, also seen in Refs. [24,26], is understood as arising from a series of multiple (experimentally unresolved) collapses [13,20,36,37,50-54], and we accordingly associate $t_{\mathrm{c}}$ with the onset of the atom loss [55]. (For further evidence for the occurrence of single and multiple discrete collapse events, see Appendix C.)

In Fig. 3(b), we show typical ToF images for different times after the quench. At $t<t_{\mathrm{c}}$, before any change in the atom number occurs, the swelling of the cloud in ToF reveals the shrinking of the wave function in-trap. Right after $t_{\mathrm{c}}$, within the first $\approx 10 \mathrm{~ms}$, we observe that the remnant cloud consists of a lower-energy central part and a higher-energy shell, reminiscent of the atom bursts generated during collapse in Ref. [24]. At longer times, we observe more irregular patterns. We see a similar shell structure in images taken along a perpendicular direction, which implies that the outgoing atom shell is spherical. Based on its size in ToF, the shell expands at a rate of $\approx 2 \mathrm{~mm} / \mathrm{s}$, which is consistent with it reflecting off the trap walls and interfering with the central part of the cloud after $\approx 10 \mathrm{~ms}$.

In Fig. 3(c), we plot $t_{\mathrm{c}}$ versus $a$ for six data sets taken with different $L$ and $N$ values. We observe $t_{\mathrm{c}}$ values that vary between 3 and 400 ms. In Fig. 3(d), we show that all the data points fall onto a single universal curve if we plot the dimensionless collapse time $t_{\mathrm{c}} / \tau_{0}$ versus the reduced distance from the critical point, $\left(a-a_{\mathrm{c}}\right) / a_{\mathrm{c}} \equiv\left(\alpha-\alpha_{\mathrm{c}}\right) / \alpha_{\mathrm{c}}$. In general, $t_{\mathrm{c}}$ could also depend on $\eta$, but the universal behavior seen in Fig. 3(d) shows that this effect is negligible for our range of $\eta$, between $4 \times 10^{-5}$ and $4 \times 10^{-4}$. The solid line in Fig. 3(d) shows results of lossless GP simulations, without any free parameters; we reproduce a very similar dependence of $t_{\mathrm{c}}$ on $\alpha$, although the numerical values are systematically slightly below the experimental ones.

\section{WEAK COLLAPSE}

We now turn to the aftermath of the collapse. Since $\tilde{\psi}$ is initially normalized to unity, the fractional atom loss $\Delta N / N$ should be some universal function of $\alpha$ and $\eta$; here, $\Delta N=N-N_{\mathrm{f}}$ is the difference between the initial (precollapse) and the final (time-dependent) atom number. The counterintuitive implication of the weak-collapse theory is that $\Delta N / N$ decreases if the BEC is made more unstable, by quenching $a$ to a more negative value.

In Fig. 4, we focus on one data set, for fixed $L=30 \mu \mathrm{m}$ and $N=20.3 \times 10^{4}$. As we illustrate in the left panel of Fig. 4(a), close to the critical point, where we observe only single-collapse events, the atom loss indeed decreases with increasing $|a|$, indicating weak collapse. On the other hand, as shown in the right panel of Fig. 4(a), in the regime of 

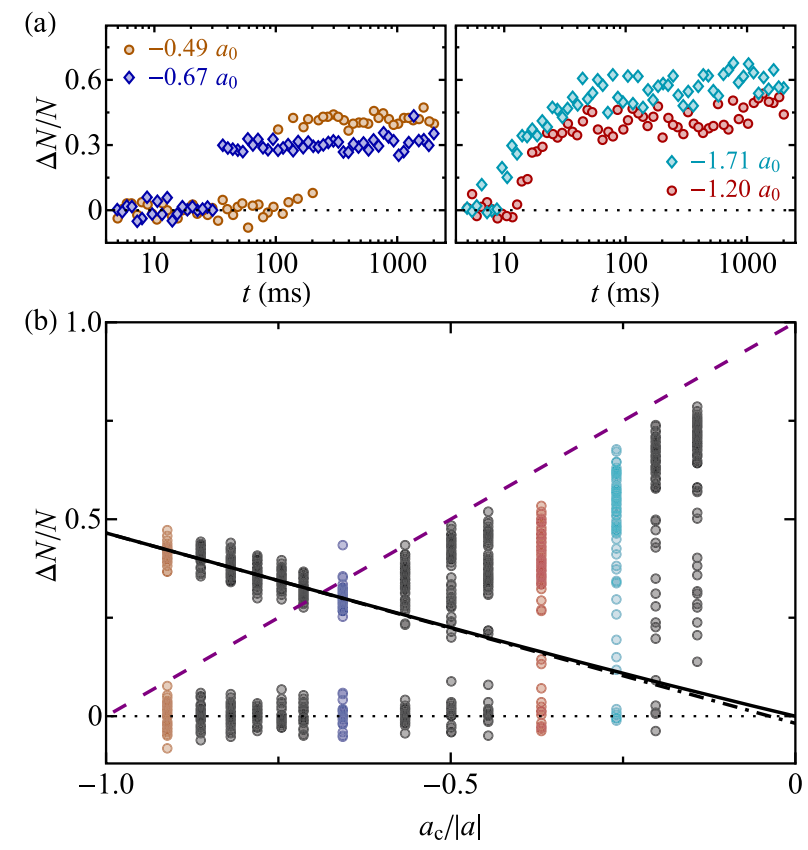

FIG. 4. Observation of weak collapse. Here $L=30 \mu \mathrm{m}$ and $N=20.3 \times 10^{4}$, corresponding to $a_{\mathrm{c}}=-0.44 a_{0}$. (a) Atom loss versus time after quenches to various $a$ values. For small $\left|a-a_{\mathrm{c}}\right|$ (left panel) $\Delta N / N$ decreases with increasing $|a|$, as expected for a weak collapse, while for large $\left|a-a_{\mathrm{c}}\right|$ (right panel) the opposite trend is seen. (b) Summary of atom loss for all $t$ and $a<a_{\mathrm{c}}$. The colored points are the data shown in (a), and the points clustered around $\Delta N=0$ correspond to $t<t_{\mathrm{c}}$. Single-collapse loss monotonically decreases with increasing $|a|$ and extrapolates to zero for $a_{\mathrm{c}} /|a| \rightarrow 0$ (dot-dashed and solid black lines, see text), confirming the prediction of the weak-collapse theory. For $a_{\mathrm{c}} /|a|>-0.6$ single collapse does not re-stabilise the system and multiple collapse occurs. The dashed purple line shows the equilibrium stability criterion, $\Delta N / N=1-a_{\mathrm{c}} / a$ (see text).

large quenches and multiple collapse, the atom loss in the long-time limit shows the opposite trend; only this type of behavior was seen in harmonic-trap experiments [24,25].

In Fig. 4(b), we present a consistent picture of the atomloss trends for all $a<a_{\mathrm{c}}$, from $a / a_{\mathrm{c}} \approx 1$ to $a / a_{\mathrm{c}} \rightarrow \infty$. Here, we plot $\Delta N / N$ versus $a_{\mathrm{c}} /|a|$, and for each $a$, we show $\Delta N / N$ values observed for all $t$; the points clustered around $\Delta N=0$ correspond to $t<t_{\mathrm{c}}$.

The single-collapse regime, $a_{\mathrm{c}} /|a|<-0.6$, is clearly identified by the small spread of the nonzero $\Delta N$ values. The single-collapse atom loss clearly decreases with increasing $|a|$ and extrapolates to zero for $a_{\mathrm{c}} /|a| \rightarrow 0$. This is the unambiguous signature of a weak collapse. The dot-dashed black line shows a linear extrapolation, which gives $\Delta N / N=-0.02(2)$ for $a_{\mathrm{c}} /|a|=0$, while the (almost indistinguishable) solid black line shows a power-law fit, $\Delta N / N \propto|a|^{-1.05(7)}$.

For $a_{\mathrm{c}} /|a|>-0.6$, multiple collapse occurs because the diminishing single-collapse atom loss does not restabilize the system. However, we see that even in this regime,

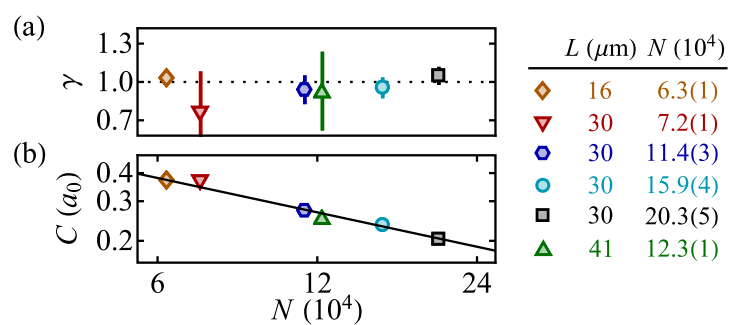

(c)

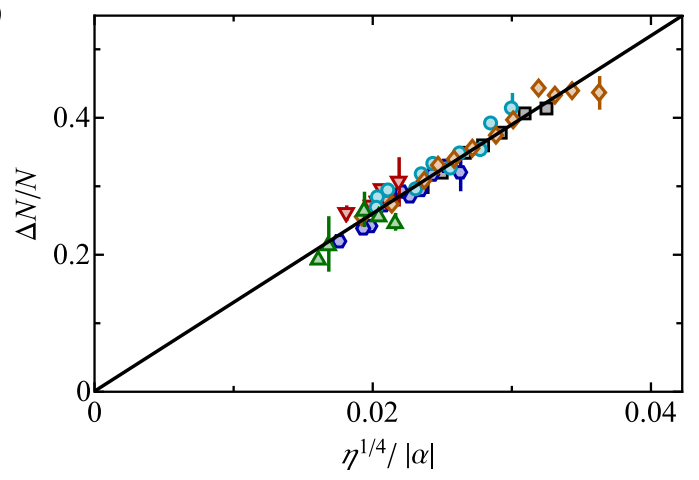

FIG. 5. Weak-collapse scaling laws. (a) Writing $\Delta N / N \propto|a|^{-\gamma}$ for fixed $L$ and $N$, for our six data sets we get an average $\bar{\gamma}=1.02(2)$. (b) Assuming $\Delta N / N=C /|a|$, we find $C \propto$ $N^{-0.51(2)} \quad$ (solid black line), with no dependence on $L$. (c) Universal behavior of the weak-collapse atom loss. We plot all the single-collapse data for different $a, N$, and $L$ versus $\eta^{1 / 4} /|\alpha|$, which vanishes in the limit of infinitely strong attractive interactions, $|\alpha| \rightarrow \infty$. The linear fit gives $\Delta N / N \approx 13 \eta^{1 / 4} /|\alpha|$.

the minimal loss we observe at each $a$ still follows the weak-collapse trend (solid black line). It is also instructive to plot the function $\Delta N / N=1-a_{\mathrm{c}} / a$ (dashed purple line); this is atom loss such that, after a quench to a given $a<a_{\mathrm{c}}$, the atom number drops to the new critical value $N_{\mathrm{c}}(a)=\alpha_{\mathrm{c}} L /(8 \pi a)=N a_{\mathrm{c}} / a$ [see Eq. (3)]. This equilibrium stability criterion is not obviously applicable in the nonequilibrium situation after the first collapse [25]. Still, it provides a good estimate of both the point, $a_{\mathrm{c}} /|a| \approx-0.6$, beyond which the single-collapse loss is insufficient to restabilize the system (see also Appendix C) and the longtime loss at large $a / a_{\mathrm{c}}$.

We now extend the study of the weak-collapse atom loss to other $L$ and $N$ values (see Fig. 5). In this analysis, we include all $a$ values for which only single collapse occurs and also those where clearly resolved single and double collapses occur (see Appendix C).

Writing $\Delta N / N \propto|a|^{-\gamma}$ for each data set with fixed $L$ and $N$, as in Fig. 4(b), we always get $\gamma$ consistent with unity [see Fig. 5(a)]; averaging over all data sets gives $\bar{\gamma}=1.02(2)$. We then assume the form $\Delta N / N=C /|a|$ and study the dependence of $C$ on $L$ and $N$. As shown in Fig. 5(b), on a log-log plot, we find $C \propto N^{-0.51(2)}$, with no clear dependence on $L$; the two points taken with $L=16 \mu \mathrm{m}$ and $41 \mu \mathrm{m}$ fall onto the same line as the four points taken with $L=30 \mu \mathrm{m}$. 
We thus experimentally find that weak-collapse atom loss is described very well by $\Delta N / N \propto 1 /(\sqrt{N}|a|)$. From Eq. (3), this corresponds to $\Delta N / N \propto \eta^{1 / 4} /|\alpha|$, which is indeed independent of $L$ and vanishes in the limit of infinitely strong attraction, $|\alpha| \rightarrow \infty$. We note that while the weak-collapse atom loss does not depend on $L$ (the overall size of the box), it may depend on the box shape; this is an interesting question for future research.

In Fig. 5(c), we plot all of our single-collapse data versus $\eta^{1 / 4} /|\alpha|$ and confirm that they fall onto a single universal curve [56]. These experimentally obtained scaling laws should provide useful input for further theoretical work.

\section{CONCLUSIONS AND OUTLOOK}

In conclusion, we have performed a comprehensive study of the collapse of an attractive BEC confined in the homogeneous potential of a 3D box trap. We have fully characterized the critical point for collapse and the collapse dynamics of an interaction-quenched BEC, finding universal behavior in agreement with the theoretical expectations. Most importantly, we have provided conclusive experimental evidence for the counterintuitive weak collapse and experimentally determined weak-collapse scaling laws that should provide a useful reference point for the general theories of nonlinear wave phenomena.

Our work also points to many avenues for further research. It would be very interesting to explore quenches from a large positive $a$, where the BEC is initially deep in the ThomasFermi regime, and in the case of a box potential, the density is uniform. In this case, it is not obvious how the condensate would spontaneously "choose" the position at which to collapse or whether many local collapses would occur instead of a global one. Additionally, since the fractional atom loss cannot exceed $100 \%$, the linear trend seen in Fig. 5(c) cannot extend to the regime of strong dissipation (large $\eta$ ). It would be interesting to explore that regime using a different geometry, a different Feshbach resonance, or a different atomic species. Finally, a major extension would be to perform similar experiments with $2 \mathrm{D}$ gases, for which a strong collapse and hence fundamentally different behavior are expected.

The data presented in this paper are available for download [57].

\section{ACKNOWLEDGMENTS}

We thank Sarah Thomas, Yago del Valle-Inclán Redondo, and Cornelius Roemer for experimental assistance, and Richard Fletcher, Raphael Lopes, and Andreas Nunnenkamp for a critical reading of the manuscript. The GeForce GTX TITAN X used for the numerical simulations was donated by the NVIDIA Corporation. This work was supported by the Royal Society, EPSRC (Grant No. EP/ N011759/1), ERC (QBox), AFOSR, and ARO. A. L. G. and N. N. acknowledge support from Trinity College, Cambridge.

\section{APPENDIX A: EXPERIMENTAL SETUP}

Our setup is the first 3D BEC box experiment with tunable interactions. The setup for producing harmonically trapped ${ }^{39} \mathrm{~K}$ condensates is similar to our previous apparatus [45]. The main difference is that here we employ the gray molasses technique [58-60] and directly cool ${ }^{39} \mathrm{~K}$ without the need for sympathetic cooling with rubidium atoms (see also Refs. [61,62]). We load the laser-cooled atoms directly into a crossed optical dipole trap (using a 20-W 1070-nm laser) and achieve efficient evaporative cooling using the Feshbach resonance in the $\left|F, m_{F}\right\rangle=$ $|1,1\rangle$ state at 402.70(3) G [63]. This results in a quasipure BEC of $\approx 2 \times 10^{5}$ atoms. We then load the atoms into a cylindrical optical box formed by blue-detuned (532-nm) laser light and cancel out gravity with a magnetic field gradient, as in Ref. [46]. The loading procedure is essentially $100 \%$ efficient and results in a quasipure box-trapped $\mathrm{BEC}$ of $\approx 2 \times 10^{5}$ atoms.

The Feshbach resonance in the $|1,1\rangle$ state has a width of $\Delta B=52 \mathrm{G}$, and the background scattering length is $a_{\mathrm{bg}}=$ $-29 a_{0}$ [64]. Hence, near the zero crossing of $a$, at $\approx 350 \mathrm{G}$, the variation of the scattering length with the magnetic field is $-a_{\mathrm{bg}} / \Delta B \approx 0.6 a_{0} / \mathrm{G}$. We tune $B$ in steps of $50 \mathrm{mG}$, corresponding to a scattering length resolution of $0.03 a_{0}$.

\section{APPENDIX B: SCATTERING LENGTH CALIBRATION}

The exact magnetic field at which the scattering length in the $|1,1\rangle$ state vanishes was independently measured in Ref. [65] to be $B_{a=0}=350.4(1)$ G. For Figs. 2(a)-2(c), we calculate our $a$ values assuming $B_{a=0}=350.4 \mathrm{G}$. Fitting the data in Fig. 2(c) with a free intercept gives an intercept $a_{\mathrm{c}}(1 / N=0)=0.03(1) a_{0}$, which is consistent with zero within the systematic $0.06 a_{0}$ error due to the uncertainty in $B_{a=0}$. We take this to be an unbiased confirmation of the zero intercept and the expected scaling $a_{\mathrm{c}} \propto 1 / N$, and we use this scaling to slightly refine the value of the zero-crossing field, to $B_{a=0}=350.45(3) \mathrm{G}$. The remaining 30-mG uncertainty in $B_{a=0}$ corresponds to a systematic uncertainty in our $a$ values of $\approx 0.02 a_{0}$.

For our interaction quenches, we have determined, using radio-frequency spectroscopy, that the magnetic field takes $4 \mathrm{~ms}$ to change (from $20 \%$ to $80 \%$ of the jump). We account for this delay in our determination of the collapse time and also include an additional 2-ms uncertainty in all the reported $t_{\mathrm{c}}$ values.

\section{APPENDIX C: FROM SINGLE TO DOUBLE COLLAPSE}

In Fig. 6, we present evidence for a gradual transition between single- and double-collapse events, which strongly supports the interpretation that an increasing number of 
(a)
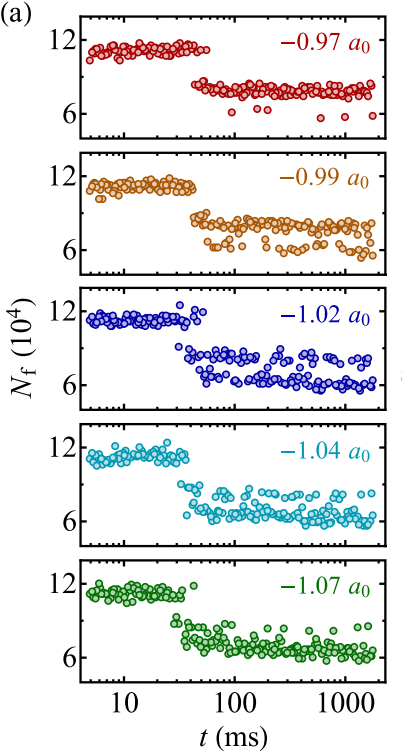

(b)

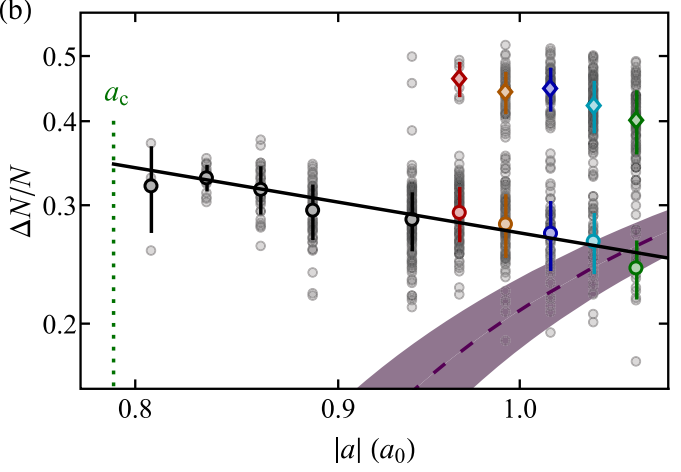

FIG. 6. Transition from one to two collapse events. (a) Atom number versus $t$ for $L=30 \mu \mathrm{m}$, initial $N=11.4 \times 10^{4}$, and various closely spaced $a$ values; here, $a_{\mathrm{c}}=-0.79 a_{0}$. On the right, we show histograms of $N_{\mathrm{f}}$ values. For $t>t_{\mathrm{c}}$, we see two clearly resolved $N_{\mathrm{f}}$ branches, corresponding to one (upper branch) and two (lower branch) collapse events. The probability of a double collapse gradually increases with $|a|$. (b) Fractional atom loss versus $|a|$ on a log-log plot. The transparent black circles show the raw data. The colored circles and diamonds show, respectively, the average values for the single- and doublecollapse events. The color code is the same as in (a). The error bars show the standard deviations. The purple dashed line shows the BEC stability criterion as in Fig. 4(b), and the shading shows its uncertainty. The solid black line shows the (single-event) weak-collapse scaling, $\Delta N / N \propto 1 /|a|$.

discrete collapse events occur as $|a|$ is increased. These data were taken with $L=30 \mu \mathrm{m}$ and initial $N=11.4 \times 10^{4}$.

In Fig. 6(a), we show the evolution of $N_{\mathrm{f}}$ after a quench to various $a<a_{\mathrm{c}}$. A fine scan of $a$ resolves a striking bifurcation of the collapse outcome. We interpret the upper and lower branches as the result of, respectively, one and two collapse events. As $|a|$ is increased, the probability of a double collapse gradually increases. This crossover is highlighted in the histograms shown on the right.

In Fig. 6(b), we show the fractional atom loss versus $|a|$ on a log-log plot. In the regime where a double collapse occurs, the single-collapse branch still clearly follows the weak-collapse scaling $\Delta N / N \propto 1 /|a|$. Note that in this data set, the double collapse occurs slightly closer to $a_{\mathrm{c}}$ than expected from the simple equilibrium stability criterion (purple band).

[1] C. Sulem and P.-L. Sulem, The Nonlinear Schrödinger Equation, Self-Focusing and Wave Collapse (SpringerVerlag, New York, 1999).

[2] G. Fibich, The Nonlinear Schrödinger Equation (Springer International Publishing, Switzerland, 2015).

[3] G. A. Askar'yan, Effects of the Gradient of a Strong Electromagnetic Beam on Electrons and Atoms, Sov. Phys. JETP 15, 1088 (1962).

[4] R. Y. Chiao, E. Garmire, and C. H. Townes, Self-Trapping of Optical Beams, Phys. Rev. Lett. 13, 479 (1964).

[5] P. L. Kelley, Self-Focusing of Optical Beams, Phys. Rev. Lett. 15, 1005 (1965).

[6] N. F. Pilipetskii and A. R. Rustamov, Observation of SelfFocusing of Light in Liquids, JETP Lett. 2, 55 (1965).

[7] M. Hercher, Laser-Induced Damage in Transparent Media, J. Opt. Soc. Am. 54, 563 (1964).

[8] V. E. Zakharov, Collapse of Langmuir Waves, Sov. Phys. JETP 35, 908 (1972).

[9] A. Y. Wong and P. Y. Cheung, Three-Dimensional SelfCollapse of Langmuir Waves, Phys. Rev. Lett. 52, 1222 (1984).

[10] P. A. Ruprecht, M. J. Holland, K. Burnett, and M. Edwards, Time-Dependent Solution of the Nonlinear Schrödinger Equation for Bose-Condensed Trapped Neutral Atoms, Phys. Rev. A 51, 4704 (1995).

[11] Y. Kagan, G. V. Shlyapnikov, and J. T. M. Walraven, BoseEinstein Condensation in Trapped Atomic Gases, Phys. Rev. Lett. 76, 2670 (1996).

[12] Y. Kagan, E. L. Surkov, and G. V. Shlyapnikov, Evolution and Global Collapse of Trapped Bose Condensates under Variations of the Scattering Length, Phys. Rev. Lett. 79, 2604 (1997).

[13] Y. Kagan, A. E. Muryshev, and G. V. Shlyapnikov, Collapse and Bose-Einstein Condensation in a Trapped Bose Gas with Negative Scattering Length, Phys. Rev. Lett. 81, 933 (1998).

[14] A. Eleftheriou and K. Huang, Instability of a Bose-Einstein Condensate with an Attractive Interaction, Phys. Rev. A 61, 043601 (2000).

[15] A. Davey and K. Stewartson, On Three-Dimensional Packets of Surface Waves, Proc. R. Soc. A 338, 101 (1974).

[16] G. C. Papanicolaou, C. Sulem, P.-L. Sulem, and X. P. Wang, The Focusing Singularity of the Davey-Stewartson Equations for Gravity-Capillary Surface Waves, Physica (Amsterdam) 72D, 61 (1994).

[17] V. E. Zakharov and V.S. Synakh, The Nature of the SelfFocusing Singularity, Sov. Phys. JETP 41, 465 (1975). 
[18] V. E. Zakharov, E. A. Kuznetsov, and S. L. Musher, Semiclassical Regime of a Three-Dimensional Wave Collapse, JETP Lett. 41, 154 (1985).

[19] V.E. Zakharov and E. A. Kuznetsov, A Quasi-Classical Theory for a Three-Dimensional Wave Collapse, Sov. Phys. JETP 64, 773 (1986).

[20] L. Bergé and J. Juul Rasmussen, Collapsing Dynamics of Attractive Bose-Einstein Condensates, Phys. Lett. A 304, 136 (2002).

[21] C. Chin, R. Grimm, P. Julienne, and E. Tiesinga, Feshbach Resonances in Ultracold Gases, Rev. Mod. Phys. 82, 1225 (2010).

[22] J. M. Gerton, D. Strekalov, I. Prodan, and R. G. Hulet, Direct Observation of Growth and Collapse of a BoseEinstein Condensate with Attractive Interactions, Nature (London) 408, 692 (2000).

[23] J. L. Roberts, N. R. Claussen, S. L. Cornish, E. A. Donley, E. A. Cornell, and C. E. Wieman, Controlled Collapse of a Bose-Einstein Condensate, Phys. Rev. Lett. 86, 4211 (2001).

[24] E. A. Donley, N. R. Claussen, S. L. Cornish, J. L. Roberts, E. A. Cornell, and C. E. Wieman, Dynamics of Collapsing and Exploding Bose-Einstein Condensates, Nature (London) 412, 295 (2001).

[25] S. L. Cornish, S. T. Thompson, and C. E. Wieman, Formation of Bright Matter-Wave Solitons During the Collapse of Attractive Bose-Einstein Condensates, Phys. Rev. Lett. 96, 170401 (2006).

[26] P. A. Altin, G. R. Dennis, G. D. McDonald, D. Döring, J. E. Debs, J. D. Close, C. M. Savage, and N. P. Robins, Collapse and Three-Body Loss in a ${ }^{85} \mathrm{Rb}$ Bose-Einstein Condensate, Phys. Rev. A 84, 033632 (2011).

[27] R. L. Compton, Y.-J. Lin, K. Jiménez-García, J. V. Porto, and I. B. Spielman, Dynamically Slowed Collapse of a Bose-Einstein Condensate with Attractive Interactions, Phys. Rev. A 86, 063601 (2012).

[28] Other experiments have explored amplification of local instabilities [29] and the $d$-wave collapse driven by dipolar interactions [30-35].

[29] J. K. Chin, J. M. Vogels, and W. Ketterle, Amplification of Local Instabilities in a Bose-Einstein Condensate with Attractive Interactions, Phys. Rev. Lett. 90, 160405 (2003).

[30] T. Lahaye, J. Metz, B. Fröhlich, T. Koch, M. Meister, A. Griesmaier, T. Pfau, H. Saito, Y. Kawaguchi, and M. Ueda, $d$-Wave Collapse and Explosion of a Dipolar Bose-Einstein Condensate, Phys. Rev. Lett. 101, 080401 (2008).

[31] T. Koch, T. Lahaye, J. Metz, B. Fröhlich, A. Griesmaier, and T. Pfau, Stabilizing a Purely Dipolar Quantum Gas Against Collapse, Nat. Phys. 4, 218 (2008).

[32] K. Aikawa, A. Frisch, M. Mark, S. Baier, A. Rietzler, R. Grimm, and F. Ferlaino, Bose-Einstein Condensation of Erbium, Phys. Rev. Lett. 108, 210401 (2012).

[33] H. Kadau, M. Schmitt, M. Wenzel, C. Wink, T. Maier, I. Ferrier-Barbut, and T. Pfau, Observing the Rosensweig Instability of a Quantum Ferrofluid, Nature (London) 530, 194 (2016).

[34] L. Chomaz, S. Baier, D. Petter, M. J. Mark, F. Wächtler, L. Santos, and F. Ferlaino, Quantum-Fluctuation-Driven Crossover from a Dilute Bose-Einstein Condensate to a
Macro-Droplet in a Dipolar Quantum Fluid, Phys. Rev. X 6, 041039 (2016).

[35] M. Schmitt, M. Wenzel, F. Böttcher, I. Ferrier-Barbut, and T. Pfau, Self-Bound Droplets of a Dilute Magnetic Quantum Liquid, Nature (London) 539, 259 (2016).

[36] L. Santos and G. V. Shlyapnikov, Collapse Dynamics of Trapped Bose-Einstein Condensates, Phys. Rev. A 66, 011602 (2002).

[37] H. Saito and M. Ueda, Mean-Field Analysis of Collapsing and Exploding Bose-Einstein Condensates, Phys. Rev. A 65, 033624 (2002).

[38] S. K. Adhikari, Dynamics of Collapsing and Exploding Bose-Einstein Condensate, Phys. Lett. A 296, 145 (2002).

[39] C. M. Savage, N. P. Robins, and J. J. Hope, Bose-Einstein Condensate Collapse: A Comparison Between Theory and Experiment, Phys. Rev. A 67, 014304 (2003).

[40] S. Métens, G. Dewel, and P. Borckmans, Nonadiabatic Effects in the Dynamics of Collapsing Bose-Einstein Condensates, Phys. Rev. A 68, 045601 (2003).

[41] M. Ueda and H. Saito, A Consistent Picture of a Collapsing Bose-Einstein Condensate, J. Phys. Soc. Jpn. 72, 127 (2003).

[42] L. D. Carr and J. Brand, Spontaneous Soliton Formation and Modulational Instability in Bose-Einstein Condensates, Phys. Rev. Lett. 92, 040401 (2004).

[43] S. Wüster, J. J. Hope, and C. M. Savage, Collapsing BoseEinstein Condensates Beyond the Gross-Pitaevskii Approximation, Phys. Rev. A 71, 033604 (2005).

[44] G. Roati, M. Zaccanti, C. D'Errico, J. Catani, M. Modugno, A. Simoni, M. Inguscio, and G. Modugno, ${ }^{39} \mathrm{~K}$ BoseEinstein Condensate with Tunable Interactions, Phys. Rev. Lett. 99, 010403 (2007).

[45] R. L. D. Campbell, R. P. Smith, N. Tammuz, S. Beattie, S. Moulder, and Z. Hadzibabic, Efficient Production of Large ${ }^{39} \mathrm{~K}$ Bose-Einstein Condensates, Phys. Rev. A 82, 063611 (2010).

[46] A. L. Gaunt, T. F. Schmidutz, I. Gotlibovych, R. P. Smith, and Z. Hadzibabic, Bose-Einstein Condensation of Atoms in a Uniform Potential, Phys. Rev. Lett. 110, 200406 (2013).

[47] Z. Shotan, O. Machtey, S. Kokkelmans, and L. Khaykovich, Three-Body Recombination at Vanishing Scattering Lengths in an Ultracold Bose Gas, Phys. Rev. Lett. 113, 053202 (2014).

[48] S. Lepoutre, L. Fouche, A. Boisse, G. Berthet, G. Salomon, A. Aspect, and T. Bourdel, Production of Strongly Bound ${ }^{39} \mathrm{~K}$ Bright Solitons, arXiv:1609.01560.

[49] M. Fattori, C. D'Errico, G. Roati, M. Zaccanti, M. JonaLasinio, M. Modugno, M. Inguscio, and G. Modugno, Atom Interferometry with a Weakly Interacting Bose-Einstein Condensate, Phys. Rev. Lett. 100, 080405 (2008).

[50] V. M. Malkin, Bi-Self-Similar Wave Collapse, JETP Lett. 48, 653 (1988).

[51] S. N. Vlasov, L. V. Piskunova, and V. I. Talanov, ThreeDimensional Wave Collapse in a Model of the Nonlinear Schrödinger Equation, Sov. Phys. JETP 68, 1125 (1989).

[52] V. E. Zakharov, N. E. Kosmatov, and V. F. Shvets, Ultrastrong Wave Collapse, Sov. Phys. JETP 49, 492 (1989).

[53] H. Saito and M. Ueda, Intermittent Implosion and Pattern Formation of Trapped Bose-Einstein Condensates with an Attractive Interaction, Phys. Rev. Lett. 86, 1406 (2001). 
[54] H. Saito and M. Ueda, Power Laws and Collapsing Dynamics of a Trapped Bose-Einstein Condensate with Attractive Interactions, Phys. Rev. A 63, 043601 (2001).

[55] Note that, in general, $t_{\mathrm{c}}$ is best defined as the time when the maximum density is reached and the atom loss rate is the highest. In all our data sets, we observe the highest loss rate at the onset of atom loss.

[56] Here, each point and its error bar show the average and standard error for a cluster of single-collapse points taken for same $\{a, N, L\}$ and just different $t>t_{\mathrm{c}}$, such as seen in Fig. 4(b) for $a_{\mathrm{c}} /|a|<-0.6$.

[57] R. Smith, C. Eigen, A. Gaunt, A. Suleymanzade, N. Navon, and Z. Hadzibabic, Research Data Supporting "Observation of Weak Collapse in a Bose-Einstein Condensate”, https://doi.org/10.17863/CAM.6607.

[58] D. Boiron, A. Michaud, P. Lemonde, Y. Castin, C. Salomon, S. Weyers, K. Szymaniec, L. Cognet, and A. Clairon, Laser Cooling of Cesium Atoms in Gray Optical Molasses down to $1.1 \mu \mathrm{K}$, Phys. Rev. A 53, R3734 (1996).

[59] G. Salomon, L. Fouché, P. Wang, A. Aspect, P. Bouyer, and T. Bourdel, Gray-Molasses Cooling of ${ }^{39} \mathrm{~K}$ to a High PhaseSpace Density, Europhys. Lett. 104, 63002 (2013).
[60] D. Nath, R. K. Easwaran, G. Rajalakshmi, and C. S. Unnikrishnan, Quantum-Interference-Enhanced Deep Sub-Doppler Cooling of ${ }^{39} \mathrm{~K}$ Atoms in Gray Molasses, Phys. Rev. A 88, 053407 (2013).

[61] M. Landini, S. Roy, G. Roati, A. Simoni, M. Inguscio, G. Modugno, and M. Fattori, Direct Evaporative Cooling of ${ }^{39} \mathrm{~K}$ Atoms to Bose-Einstein Condensation, Phys. Rev. A 86, 033421 (2012).

[62] G. Salomon, L. Fouché, S. Lepoutre, A. Aspect, and T. Bourdel, All-Optical Cooling of ${ }^{39} \mathrm{~K}$ to Bose-Einstein Condensation, Phys. Rev. A 90, 033405 (2014).

[63] R. J. Fletcher, R. Lopes, J. Man, N. Navon, R. P. Smith, M. W. Zwierlein, and Z. Hadzibabic, Two and Three-Body Contacts in the Unitary Bose Gas, arXiv:1608.04377.

[64] C. D'Errico, M. Zaccanti, M. Fattori, G. Roati, M. Inguscio, G. Modugno, and A. Simoni, Feshbach Resonances in Ultracold ${ }^{39}$ K, New J. Phys. 9, 223 (2007).

[65] M. Fattori, G. Roati, B. Deissler, C. D’Errico, M. Zaccanti, M. Jona-Lasinio, L. Santos, M. Inguscio, and G. Modugno, Magnetic Dipolar Interaction in a Bose-Einstein Condensate Atomic Interferometer, Phys. Rev. Lett. 101, 190405 (2008). 